This item was submitted to Loughborough's Research Repository by the author.

Items in Figshare are protected by copyright, with all rights reserved, unless otherwise indicated.

\title{
Low-frequency ground vibrations from underground trains
}

PLEASE CITE THE PUBLISHED VERSION

http://www.multi-science.co.uk/lowfreq.htm

PUBLISHER

(C) Multi-Science Publishing

VERSION

VoR (Version of Record)

LICENCE

CC BY-NC-ND 4.0

REPOSITORY RECORD

Krylov, Victor V.. 2012. "Low-frequency Ground Vibrations from Underground Trains". figshare. https://hdl.handle.net/2134/10062. 
This item was submitted to Loughborough's Institutional Repository (https://dspace.lboro.ac.uk/) by the author and is made available under the following Creative Commons Licence conditions.

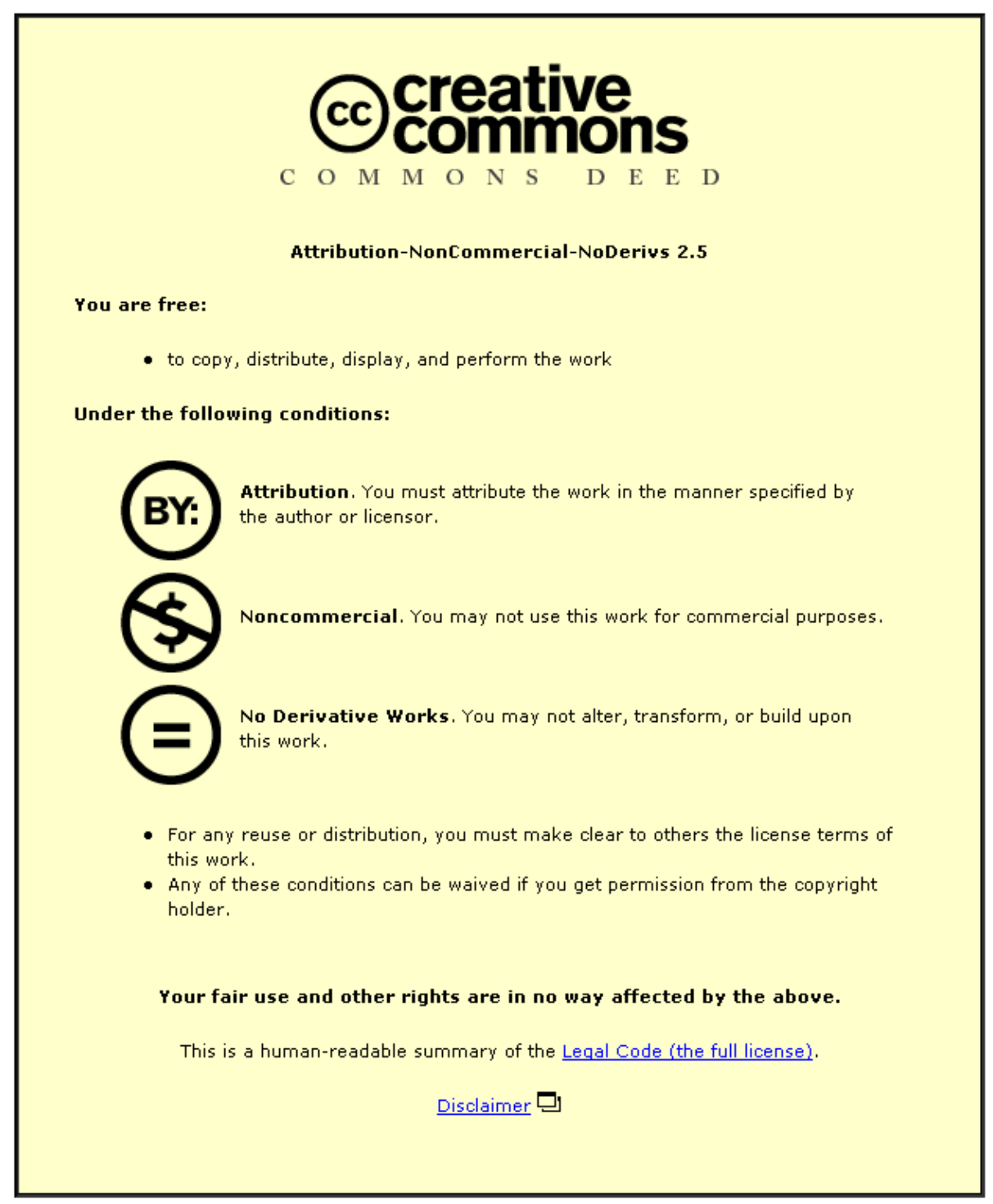

For the full text of this licence, please go to: http://creativecommons.org/licenses/by-nc-nd/2.5/ 
Low-Frequency Ground Vibrations from

Underground Trains

by

\author{
V.V. Krylov \\ Reprinted from \\ JOURNAL OF \\ LOW FREQUENCY \\ NOISE \& VIBRATION
}

VOLUME 14 No. 11995

MULTI-SCIENCE PUBLISHING CO. LTD.

107 High Street, Brentwood, Essex CM14 4RX, United Kingdom 


\title{
Low-Frequency Ground Vibrations from Underground Trains
}

\author{
V.V. Krylov \\ Centre for Research into the Built Environment, Nottingham Trent University, \\ Burton Street, Nottingham NG1 4BU, UK
}

\begin{abstract}
In the present work, the ground vibrations generated by underground trains are investigated theoretically in the low-frequency approximation, i.e. for characteristic wave-lengths of generated bulk acoustic waves in the ground being essentially larger than the diameter of the tunnel. The Green's function formalism is applied to calculate contributions of all sleepers of the track located inside the tunnel and subjected to the action of a quasi-static pressure from all wheel axles. Parameters of a train, track and a tunnel as well as mechanical properties of soil (including contact nonlinearity of the track-soil system) are being taken into account. The corresponding numerical calculations show that for most of the practical values of a tunnel depth, the main contribution to the vertical component of the surface ground vibration velocity is due to the radiated shear bulk waves rather than to the longitudinal ones. Comparison of the ground vibration spectra generated by typical underground trains with the spectra of the above-ground trains shows that the shapes of these spectra are similar. This implies that one can use for underground trains the same methods of suppressing ground vibrations at speed-dependent frequencies that can be used for above-ground trains, e.g., by choosing special relations between the track and train parameters.
\end{abstract}

\section{INTRODUCTION}

Railways, which were considered an anachronism during the last two decades, are now experiencing dramatic revival in Europe. They have suddenly become one of the most advanced and fast developing branches of transportation technology that may be compared with the space technology breakthrough of the sixties. The reason is the high speeds achievable by the most advanced modern railway trains. Speeds close to or greater than $300 \mathrm{~km} / \mathrm{h}$ are now quite typical for French TGV-trains and German ICE-trains. British Rail is also in a position to introduce high-speed services on lines linked with the continent through the Channel Tunnel.

The increased attention to railway communications makes it especially important to predict the environmental impact of railway trains, including trains travelling underground. In contrast to the above-ground trains for which the direct railway noise propagating through the air inside the buildings is usually more intensive than structure-borne noise associated with ground vibrations (ground elastic waves), in the case of underground trains, the ground-borne noise makes the main contribution. Understanding basic mechanisms of generating ground vibrations by underground trains is of particular importance in constructing new underground railway-lines since major environmental concerns of residents about new underground lines are usually connected with expected vibrations and structure-borne noise, as well as with possible damage to their properties. Both these effects depend strongly on the spectra of 
generated ground vibrations that, in turn, are functions of the train, track and tunnel parameters as well as of soil properties.

Several experimental investigations of ground vibration spectra from underground railways have been carried out (e.g., Refs. 1 \& 2). However, apart from the papers 3,4 using an indirect impedance approach to estimate the energy of ground vibrations generated by underground trains, no theoretical investigation based on analytical solution of the elastic field equations has been undertaken so far.

In the present work, the approximate low-frequency analysis is attempted using the approach recently developed at the Nottingham Trent University for above-ground trains 5,6 . According to this approach, the Green's function formalism is applied to calculate ground vibrations generated by all sleepers of the track located inside the tunnel and subjected to the action of a quasi-static pressure from all wheel axles. For high quality wheels and tracks, this excitation mechanism gives the major contribution to the ground vibration spectra in the low-frequency range (below $50 \mathrm{~Hz}$ ). Parameters of a train, track and a tunnel as well as mechanical properties of soil (including contact nonlinearity of the track-soil system) are taken into account. The main practical aim of this study is to explore methods to suppress ground vibrations as much as possible on the track itself.

\section{THEORETICAL BACKGROUND}

In comparison with above-ground trains, the case of underground trains is more complicated for theoretical description, partly because of the influence of the tunnel geometry making the problem of constructing the corresponding Green's function extremely difficult. We recall that the physical meaning of the Green's function for the problem under consideration is that it describes the ground vibrations generated by an individual sleeper which may be regarded as a point source in the low-frequency band. In the case of underground trains, i.e. for sleepers placed on the bottom of a tunnel, bulk acoustic waves usually make a major contribution to the ground vibration field near the surface, in contrast to the case of above-ground trains where Rayleigh surface acoustic waves prevail.

The approximate analytical approach being described here considers the problem in the low-frequency approximation, i.e. the characteristic wavelengths of generated bulk acoustic waves in the ground are assumed to be essentially larger than the diameter of the tunnel (Figure 1). In such an approximation the formal expression for the vertical component of the particle velocity of ground vibrations generated on the ground surface by trains travelling underground may be written as follows:

$$
v_{z}(x, y, \omega)=\int_{-\infty}^{\infty} \int_{-\infty}^{\infty} \int_{-\infty}^{\infty} P\left(x^{\prime}, y^{\prime}, z^{\prime}, \omega\right) G_{z z}(r, \omega) d x^{\prime} d y^{\prime} d z^{\prime}
$$

Here $G_{z z}(r, \omega)$ is the correspondent component of the elastic half-space Green's tensor (Green's function) describing the vertical component of the particle vibration velocity due to a vertical point force located in the depth of the elastic medium, $r=\left[\left(x-x^{\prime}\right)^{2}+\left(y-y^{\prime}\right)^{2}+\left(z-z^{\prime}\right)^{2}\right]^{1 / 2}$ is the distance from the current elementary source to the observation point, and $\mathrm{P}\left(\mathrm{x}^{\prime}, \mathrm{y}^{\prime}, \mathrm{z}^{\prime}, \omega\right)$ describes the Fourier transform of the total distribution of vertical load forces along the underground track.

For large distances $r$ (in comparison with wave-lengths of radiated waves) the approximate expression for the Green's function $G_{z z}(r, \omega)$ at $z=0$ may be written with only bulk elastic waves being taken into account.

$$
\begin{aligned}
& \mathrm{G}_{\mathrm{zz}}(\mathrm{r}, \omega) \mathrm{I}_{\mathrm{z}=0}=\left(\mathrm{i} \omega / 4 \pi \rho_{0} \mathrm{r}\right)\left[\mathrm{e}_{1}^{\mathrm{i}(\omega / \mathrm{c})}\left(1+\mathrm{R}_{\mathrm{l}}(\omega, \varphi)\right) \cos ^{2}(\varphi)-\right. \\
&\left.\mathrm{e}_{\mathrm{t}}^{\mathrm{i}(\omega / \mathrm{c})}\left(1+\mathrm{R}_{\mathrm{t}}(\omega, \varphi)\right) \sin ^{2}(\varphi)\right]
\end{aligned}
$$


Here terms with the exponents $\mathrm{e}^{\mathrm{i}(\omega / \mathrm{c})}$ and $\mathrm{e}^{\mathrm{i}(\omega / \mathrm{c})}$ describe contributions of radiated longitudinal and shear bulk waves, $R_{l}(\omega, \varphi)$ and $R_{t}(\omega, \varphi)$ are the corresponding reflection coefficients from the surface for the incident longitudinal and shear waves respectively (note that each of these coefficients takes account of both waves reflected from the surface - longitudinal and shear), and $\varphi$ is the observation angle relative to the vertical direction $(\cos \varphi=$ $\left.\left(z-z^{\prime}\right) / r\right)$. The dependence on frequency in $R_{l}(\omega, \varphi)$ and $R_{t}(\omega, \varphi)$ takes account of impedance load resulting from the influence of buildings or other engineering structures on the surface. In what follows we assume, without loss of generality, that $R_{l}(\omega, \varphi)=0$ and $R_{t}(\omega, \varphi)=0$, i.e. all the energy of radiated waves is absorbed by the structure.

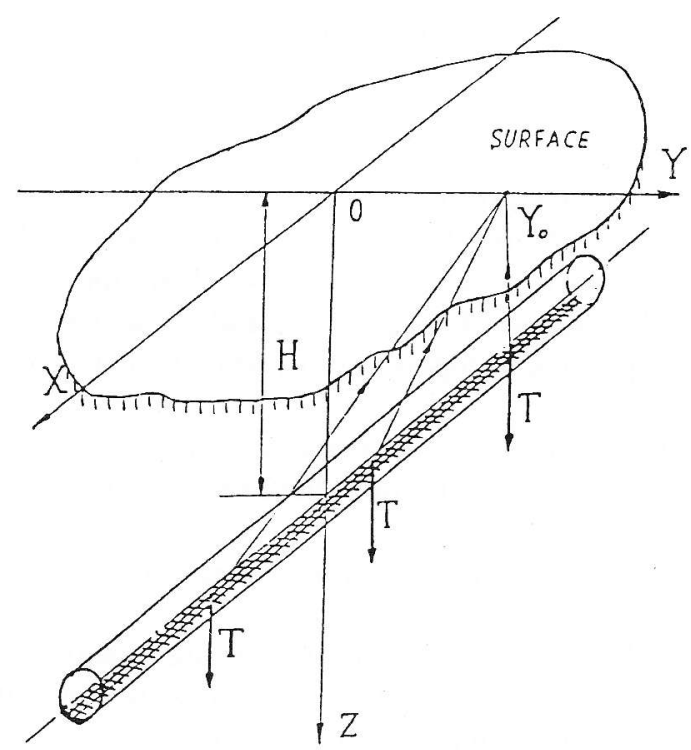

Figure 1. Geometry of the problem.

The load-force distributions along underground tracks may be written in the form similar to that for the above-ground tracks 5 . For a full train moving at speed $v$ along the track lying underground at the depth $\mathrm{H}$ this distribution has the form

$$
\begin{gathered}
P\left(t, x^{\prime}, y^{\prime}, z^{\prime}\right)=\sum_{m=-\infty}^{\infty} \sum_{n=0}^{N-1} A_{n}\left[P\left(t-\left(x^{\prime}+n L\right) / v\right)+\right. \\
\left.P\left(t-\left(x^{\prime}+M+n L\right) / v\right)\right] \delta\left(x^{\prime}-m d\right) \delta\left(y^{\prime}\right) \delta\left(z^{\prime}-H\right)
\end{gathered}
$$

Here $\mathrm{m}$ denotes a number of a current sleeper, $\mathrm{n}$ denotes a number of a current carriage, $\mathrm{N}$ is the number of carriages, $\mathrm{M}$ is the distance between bogies in a carriage, $L$ is the total carriage length, $A_{n}$ takes account of different carriage masses (in further consideration we assume for simplicity that all masses are equal, i.e. $\left.A_{n}=1\right) ; P\left(t-x^{\prime} / v\right)$ is determined by the expression

$$
P\left(t-x^{\prime} / v\right)=T\left[2 w\left(t-x^{\prime} / v\right) / w_{\max }\right]\left(d / x_{0}\right)
$$

where $\mathrm{T}$ is the axle load, $\mathrm{w}_{\max }$ is the maximum value of the track deflection function $\mathrm{w}(\mathrm{x})=\mathrm{w}(\mathrm{vt})$ resulting from the quasi-static pressure of the moving axle load, $\mathrm{d}$ is the distance between adjacent sleepers, and $\mathrm{x}_{0}$ is the characteristic deflection distance (terms on the right of $\mathrm{T}$ take into account the distribution of axle load between sleepers within the deflection distance ${ }^{5}$. 
Taking the Fourier transform of Eqn. (3) and substituting it into Eqn. (1) with account of (2) and (4), one can obtain, after some manipulations, the following expression for the frequency spectra of vertical vibrations at $z=0, x=0$ and $\mathrm{y}=\mathrm{y}_{0}$ generated by an underground train:

$$
\begin{gathered}
v_{z}\left(0, y_{0}, \omega\right)=\left[i \omega P(\omega) / 4 \pi \rho_{0}\right] \sum_{m=-\infty}^{\infty} \sum_{n=0}^{N-1}[1+\exp (i M \omega / v)] \exp [i(\omega / v)(m d+n L)] \times \\
\left(1 / r_{m}\right)\left\{\exp \left[\left(-\gamma_{1} \omega r_{m} / c_{l}\right)+i\left(\omega / c_{1}\right) r_{m}\right] \cos ^{2}\left(\varphi_{m}\right)-\exp \left[\left(-\gamma_{t} \omega r_{m} / c_{t}\right)+\right.\right. \\
\left.\left.i\left(\omega / c_{t}\right) r_{m}\right] \sin ^{2}\left(\varphi_{m}\right)\right\}
\end{gathered}
$$

Here $r_{m}=\left[y_{0}{ }^{2}+(m d)^{2}+H^{2}\right]^{1 / 2}, \cos \left(\varphi_{m}\right)=H / r_{m}$, and $P(\omega)=(1 / 2 \pi) \int^{\infty} P(t)$ $\exp (i \omega t) d t$ is a Fourier transform of $P(t)$. In writing Eqn. (5) we account ${ }^{-\infty}$ for attenuation in soil by replacing $1 / c_{1}$ and $1 / c_{t}$ in the exponentials by the complex values $1 / c_{1}+i \gamma_{1} / c_{1}$ and $1 / c_{t}+i \gamma_{t} / c_{t}$, where $\gamma_{1, t}<<1$ are the constants describing the "strength" of dissipation of longitudinal and shear waves in the soil (Eqn. (5) implies a linear frequency dependence of soil attenuation, in agreement with experimental data).

To generalise Eqn. (5) to describe the action of two axle loads separated by the distance a (the case of a bogie) one should replace $\mathrm{P}(\omega)$ by:

$$
\mathrm{P}_{\mathrm{b}}(\omega)=2 \mathrm{P}(\omega) \cos (\omega \mathrm{a} / 2 \mathrm{v})
$$

It follows from Eqn. (5) that, similarly to the case of above-ground trains, the spectrum of ground vibrations under consideration is quasi-discrete, with the maxima at frequencies determined by the condition $(\omega / v)(s d+q L)=2 \pi l$, where $\mathrm{s}, \mathrm{q}, 1=1,2,3, \ldots$ Obviously, $\mathrm{q}=0$ corresponds to the passage frequencies $\mathrm{f}_{\mathrm{p}} \mathrm{s}$ determined by the sleeper period $d$. Other more frequent maxima are determined either by the carriage length $\mathrm{L}(\mathrm{s}=0)$ or by a combination of both parameters (for $\mathrm{q} \neq 0, \mathrm{~s} \neq 0$ ).

The summation over $\mathrm{m}$ in Eqn. (5) considers an infinite number of sleepers. However, the contribution of remote sleepers is small because of soil attenuation and spherical spreading, and a few hundred sleepers are adequate for practical calculations.

\section{NUMERICAL RESULTS AND DISCUSSION}

Numerical calculations of the ground vibration velocity according to Eqn. (5) have been carried out for different train, track, soil and tunnel parameters. It follows from the calculations that for most of the practical values of a tunnel depth $\mathrm{H}$, the main contribution to the veritcal component $\mathrm{v}_{\mathrm{z}}$ of the total ground vibration field at the ground surface is due to the radiated shear bulk waves rather than to the longitudinal bulk waves.

The amplitudes of generated ground vibrations at the frequency $15 \mathrm{~Hz}$ (in $\mathrm{dB}$ re $10^{-9} \mathrm{~m} / \mathrm{s}$ ) as functions of the tunnel depth $\mathrm{H}$ for $\mathrm{Y}_{0}=0$ are shown in Figure 2: the total field (A) and the contributions of longitudinal (B) and shear (C) waves separately. Speed of a 5 -carriage train $(\mathrm{N}=5)$ was $50 \mathrm{~km} / \mathrm{h}$; other parameters were the following: the elastic parameters of the soil were $c_{t}=272$ $\mathrm{m} / \mathrm{s}, \mathrm{c}_{1}=471 \mathrm{~m} / \mathrm{s}$ (corresponding to a Poisson's ratio of $\sigma=0.25$ ), the mass density of soil $\rho_{0}$ was set at $2000 \mathrm{~kg} / \mathrm{m}^{3}$, the axle load was $100 \mathrm{kN}$, and geometrical dimensions of the train were $\mathrm{L}=8.3 \mathrm{~m}, \mathrm{M}=4.88 \mathrm{~m}$ and $\mathrm{a}=2.2 \mathrm{~m}$. The soil attenuation parameters $\gamma_{1, \mathrm{t}}$ were chosen as 0.005 .

It is seen that for values of $H$ less than $150-160 \mathrm{~m}$ the contribution of shear waves $(\mathrm{C})$ is essentially superior to the contribution of longitudinal waves (B). 
For larger depths, the contributions of shear and longitudinal waves become comparable with each other and the resulting field (A) shows an oscillatory behaviour versus $H$.

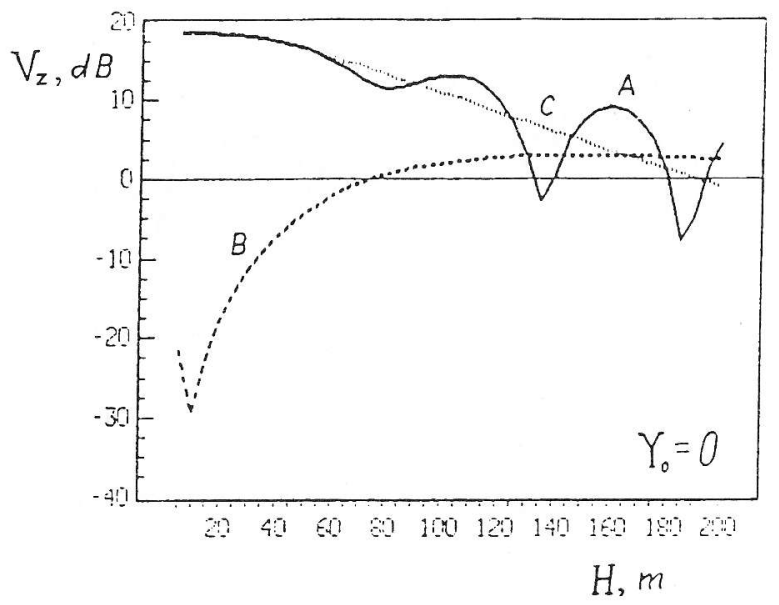

Figure 2. Ground vibration amplitudes as functions of the tunnel depth $\mathrm{H}$.

Generated ground vibrations at the frequency $15 \mathrm{~Hz}$ as functions of the observation distance from the track $Y_{0}$ for $\mathrm{H}=30 \mathrm{~m}$ are shown in Figure 3: the total field (A) and the contributions of longitudinal (B) and shear (C) waves. Parameters of the train are the same as in Figure 2. One can see that all fields decrease with the distance $Y_{0}$, especially the field of longitudinal waves (B).

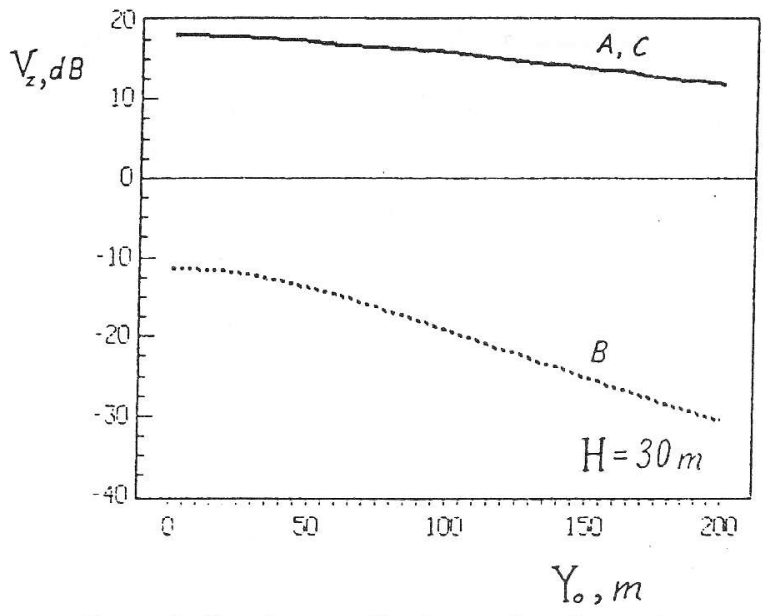

Figure 3. Ground vibration amplitudes as functions of the distance $\mathrm{Y}_{0}$.

Figure 4 illustrates full ground vibration spectra generated by the abovementioned underground trains. Calculations were carried out for the values of tunnel depth $\mathrm{H}=30,60$ and $90 \mathrm{~m}$ (curves $\mathrm{A}, \mathrm{B}$ and $\mathrm{C}$ respectively). Other parameters are the same as on the previous figures. For comparison, the corresponding spectrum of ground vibrations from the above-ground train of the same parameters is also displayed on this figure (curve D). One can see that, although lower in amplitudes, the shapes of the ground vibration spectra for underground trains are very similar to those generated by the trains travelling above the ground. This implies that one can use the same methods of suppressing ground vibrations at speed-dependent frequencies that can be used for above-ground trains ${ }^{4,5}$, e.g. by choosing special relations between the track and train parameters. 


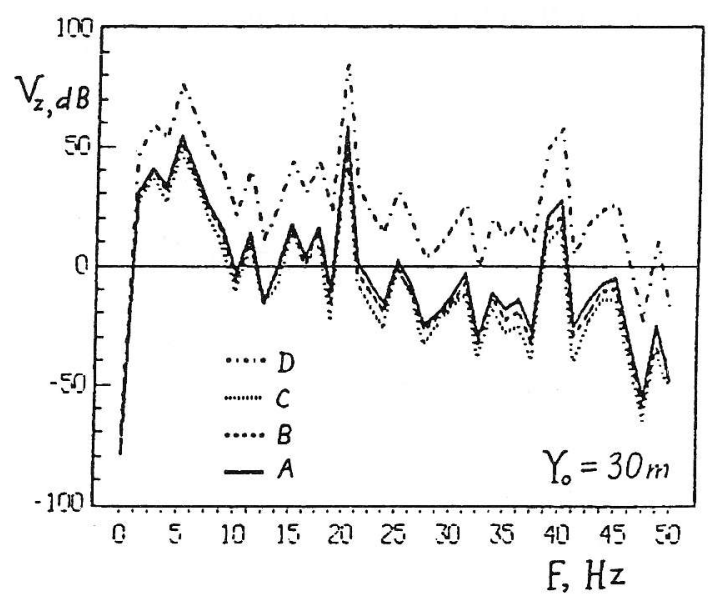

Figure 4. Ground vibration spectra for under- and above-ground trains.

\section{REFERENCES}

1. A. Trochides, Applied Acoustics, 32, 289 (1991).

2. S. Ljunggren, Applied Acoustics, 34, 193 (1991).

3. M. Heckle, et al., Report (Institut für Technische Akustik, Berlin, March 1987).

4. J. Melke, J. Sound and Vibr. 120, 391 (1988).

5. V. Krylov and C. Ferguson, Applied Acoustics, 42, 199 (1994).

6. V.V. Krylov, Journal de Physique, 4, C5-769 (1994). 